\title{
Nonlinear optical properties of $\mathrm{Au}-\mathrm{Ag}$ nanoplanets made by ion beam processing of bimetallic nanoclusters in silica
}

\author{
T. Cesca ${ }^{\text {a,* }}$, G. Pellegrini ${ }^{\text {a, }}$ V. Bello ${ }^{\text {a }}$, C. Scian ${ }^{\text {a }}$, P. Mazzoldi ${ }^{\text {a }}$, P. Calvelli ${ }^{\text {b }}$, G. Battaglin ${ }^{\text {b }}$, G. Mattei $^{\text {a }}$ \\ ${ }^{a}$ Department of Physics, University of Padova, I-35131 Padova, Italy \\ ' Department of Physical Chemistry, Ca' Foscari University of Venice, I-30123 Venice, Italy
}

\section{A R T I C L E I N F O}

\section{Article history:}

Received 16 October 2009

Available online 2 June 2010

\section{Keywords:}

Glass composites

Nanoclusters

Ion irradiation

Nonlinear optics

\begin{abstract}
A B S T R A C T
The nonlinear absorption of Au-Ag nanoplanets made by Ar irradiation of bimetallic nanoclusters in silica has been experimentally investigated by means of the single beam z-scan technique. The measurements have been performed in the picoseconds regime in order to isolate the fast electronic contribution to the third-order nonlinearity. The results reveal large nonlinear absorption properties of these systems, characterized by the concomitance of saturable and reverse saturable absorption. A phenomenological expression has been developed to fit the z-scan curves and to quantitatively determine the nonlinear optical parameters.
\end{abstract}

(c) 2010 Elsevier B.V. All rights reserved.

\section{Introduction}

Metal nanocluster composite glasses (MNCGs) have attracted much interest over the last decade for their peculiar optical, magnetic and catalytic properties $[1,2]$. The MNCGs also exhibit interesting nonlinear optical (NLO) properties, related to the strong surface plasmon resonance in the visible spectrum, which make them very promising for molecular sensing applications and as ultrafast nonlinear optical elements [2-6].

Among the different techniques, ion irradiation proved to be a very versatile tool for synthesizing and processing plasmonic nanostructures in insulating matrices. On the other hand the functional properties of the nanostructures can be properly tuned by a tailored control of the irradiation parameters [2-4]. Recently, ion irradiation has been effectively employed to produce a new class of nanocluster structures named "nanoplanets" (NPls), consisting of a central cluster surrounded by small satellites clusters [7]. The structural and compositional properties of these systems have been characterized in details as a function of the irradiation conditions (ion, energy, fluence) [8]. Moreover, the plasmonic properties of the NPls have been theoretically investigated in the framework of a full-interaction electromagnetic approach [9], revealing large local-field enhancements in the satellite cluster halos.

In this work we focused on the third-order nonlinear absorption properties of bimetallic Au-Ag nanoplanets. These properties are commonly defined in terms of an intensity-dependent absorption coefficient $\alpha(I)=\alpha_{0}+\beta I$, where $\alpha_{0}$ and $\beta$ are the linear and nonlinear absorption coefficients, respectively. The NLO response of the

\footnotetext{
* Corresponding author. Fax: +390498277003.

E-mail addresses: cesca@padova.infm.it, tiziana.cesca.1@unipd.it (T. Cesca).
}

NPls has been experimentally investigated by means of the single beam z-scan technique [10]. The measurements have been performed with a pulsed laser beam in the picoseconds regime in order to isolate the fast electronic contribution to the third-order nonlinearity. The samples exhibit promisingly large nonlinear absorption properties characterized by the concomitance of saturable (SA) and reverse saturable (RSA) absorption. This makes the evaluation of the nonlinear optical parameters not straightforward. A phenomenological function has been developed to fit the z-scan curves, to quantitatively determine the coefficient of SA and RSA.

\section{Experimental}

$\mathrm{Au}^{+}$and $\mathrm{Ag}^{+}$sequential ion implantations were performed at room temperature on fused silica (type II, Heraeus) slides with a $200 \mathrm{kV}$ high-current implanter (Danfysik 1090) at the INFM-INFN Ion Implantation Laboratory (Legnaro, Italy). The current density was maintained at $2 \mu \mathrm{A} / \mathrm{cm}^{2}$. Ion energies of $190 \mathrm{keV}$ for $\mathrm{Au}$ and $130 \mathrm{keV}$ for $\mathrm{Ag}$ were chosen in order to obtain comparable ion ranges, and the fluence was $3 \times 10^{16}$ ions $/ \mathrm{cm}^{2}$ for both ions. The implanted slides were then annealed in air at $800^{\circ} \mathrm{C}$ for $1 \mathrm{~h}$. Subsequently, Ar irradiations were performed on a set of samples at energy of $190 \mathrm{keV}$, current density of $0.2 \mu \mathrm{A} / \mathrm{cm}^{2}$ and fluence of $2.5 \times 10^{16}$ ions $/ \mathrm{cm}^{2}$.

Structural and compositional characterization was performed by transmission electron microscopy (TEM) with a field-emission gun (FEG) microscope operating at $200 \mathrm{kV}$ equipped with an EDAX energy-dispersive X-ray spectrometer (EDS). Optical absorption spectra were collected with a JASCO V670 dual beam spectrophotometer in the $300-800 \mathrm{~nm}$ wavelength range. 
The nonlinear optical properties of the samples were investigated by the z-scan technique. A ring-cavity, mode-locked Nd:glass laser was used as laser source. The laser supplies trains of about 100 pulses at a repetition rate of $1 \mathrm{~Hz}$. A pulse slicer was used to select a single pulse (about 6 ps duration) out of each train. The TEM $_{00}$ laser output is frequency doubled $(\lambda=527 \mathrm{~nm})$ by means of a second harmonic birifrangent crystal ( $\beta$-barium-borate, $\beta$ BBO). A $100 \mathrm{~mm}$ converging lens was used to focus the laser beam. The beam waist $w_{0}$ at the focal point was about $20 \mu \mathrm{m}$. Closed aperture (CA) and open aperture (OA) transmittance curves were collected simultaneously at each sample position along the beam propagation direction and normalized to a reference signal. Optical density filters were used to vary the laser intensity at the lens focus. In order to avoid sample damage or modifications during the scans, the maximum laser intensity at the beam waist used in the measurements was $I_{0}=0.8 \mathrm{GW} / \mathrm{cm}^{2}$. Each point in the z-scan curves is the average of 64 shots. $\mathrm{A} \mathrm{CS}_{2}$ reference sample $\left(n_{2}=3 \times 10^{-14} \mathrm{~cm}^{2} / \mathrm{W}\right)$ was used to calibrate the laser intensity.

\section{Results and discussion}

Bright-field cross-sectional TEM analyses of the implanted samples, before and after Ar irradiation, are reported in Fig. 1. As shown (Fig. 1a), the sequential $\mathrm{Au}^{+}, \mathrm{Ag}^{+}$implantation and the subsequent heat treatments of the samples induce the formation of a population of AuAg alloy clusters with an average diameter $\langle D\rangle=12 \pm 6 \mathrm{~nm}$, centered at about $60 \mathrm{~nm}$ below the surface. Upon Ar irradiation (Fig. 1b), then, the formation of Au-enriched satellite clusters around each original cluster is promoted, giving rise to the nanoplanets (NPls) structure [8].

The peculiar topology of the NPls controls both the linear and the nonlinear optical properties of the samples. In Fig. 2 we re-
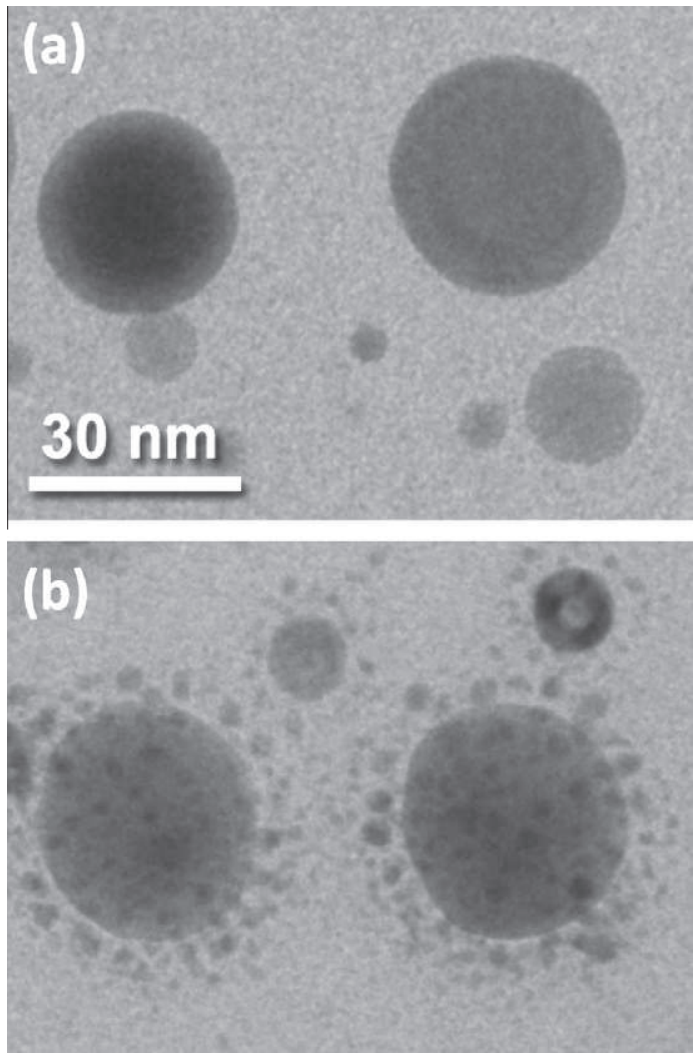

Fig. 1. TEM cross-sectional bright-field micrographs of $\mathrm{Au}^{+}, \mathrm{Ag}^{+}$implanted samples, before (a) and after (b) Ar irradiation.

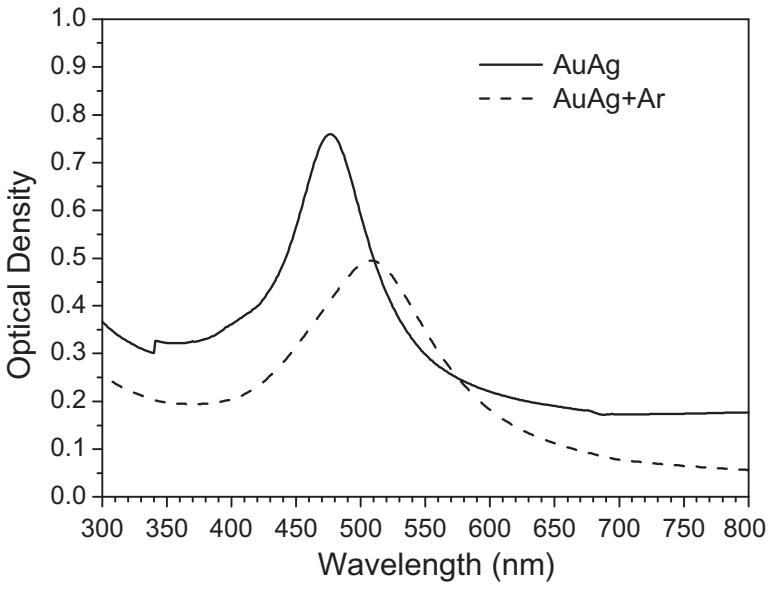

Fig. 2. Optical absorption spectra of samples AuAg (before Ar irradiation) and $\mathrm{AuAg}+\mathrm{Ar}$ (after Ar irradiation).

ported the optical absorption spectra of the samples before (AuAg) and after $(\mathrm{AuAg}+\mathrm{Ar}) \mathrm{Ar}$ irradiation. Red-shift and dumping of the SPR absorption band are visible. These have been attributed to the electromagnetic coupling between the core and the satellite nanoclusters, which affects the local field near the core surface [9]. Theoretical calculations have also revealed the formation of hot-spots with a local-field enhancement of about a factor 10 [4].

In order to investigate the influence of these effects on the nonlinear optical properties, we performed z-scan measurements on both the AuAg and the AuAg + Ar samples. For materials that exhibit NLO response, the $z$-scan technique allows to determine magnitude and sign of both the nonlinear refractive index $\left(n_{2}\right)$ and the nonlinear absorption coefficient $(\beta)$ [10]. The basic idea of this technique is to measure the transmitted intensity as the sample is moved along the propagation direction $(z)$ of a laser beam focused by a converging lens. In this way, the intensity on the sample varies as a function of $z$, being maximum at the lens focus, and the nonlinear effects can be detected as variation in the normalized transmittance. In particular closed aperture (CA) transmittance curves measured through a small aperture are sensitive to phase distortions and are affected by both nonlinear refraction and nonlinear absorption, whereas open aperture $(\mathrm{OA})$ measurements without the aperture carry information only on the nonlinear absorption effects. A schematic diagram of the z-scan set-up is reported in Fig. 3.

In the conventional approach developed by Sheik-Bahae for a Gaussian laser beam [10], the OA z-scan results can be fitted with an analytical function $T_{\mathrm{OA}}(z)$ to extract the nonlinear absorption coefficient $\beta$ :

$T_{O A}=1-\frac{\beta I_{0} L_{\mathrm{eff}}}{2 \sqrt{2}\left[1+\left(\frac{z}{z_{0}}\right)^{2}\right]}$

where $I_{0}$ is the peak laser intensity at the lens focus and $z_{0}$ is the diffraction length (Rayleigh range) of the focused Gaussian beam. $L_{\text {eff }}=\frac{1-e^{-\alpha_{0} L}}{\alpha_{0}}$ is the sample effective length and it is function of the sample thickness $L$ and linear absorption coefficient at the laser wavelength, $\alpha_{0}$. From Eq. (1), a positive nonlinear absorption $(\beta>0)$ corresponds to a decrease of the transmittance as the sample approaches the lens focus $(z=0)$ and appears as a minimum in the OA z-scan curve. Such phenomenon is commonly referred to as reverse saturable absorption (RSA). Conversely, a negative nonlinear absorption coefficient $(\beta<0)$ gives rise to an increase of $T_{\mathrm{OA}}$ and produces a peak in the transmittance curve. In this case the material exhibits saturable absorption (SA). 


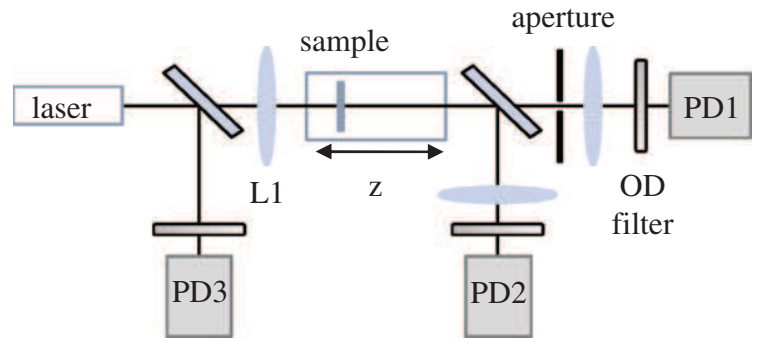

Fig. 3. Schematic diagram of the $\mathrm{z}$-scan set-up. $\mathrm{L} 1$ is the $100 \mathrm{~mm}$ converging lens used to focus the laser beam. Closed aperture and open aperture transmittance curves are detected simultaneously by photodetectors PD1 and PD2, and normalized to the reference signal detected by PD3.

Fig. 4 shows the OA transmittance curves of the samples, measured with a laser intensity at the lens focus $I_{0}=0.1 \mathrm{GW} / \mathrm{cm}^{2}$. The curves are normalized to the linear transmittance measured far from the focus. The OA scan of sample AuAg (Fig. 4a) shows a deep minimum at the lens focus $(z=0)$, characteristic of a positive value of the nonlinear absorption coefficient $\beta$. The best fit of the data in the Sheik-Bahae frame is shown as dashed line in Fig. 4a. The nonlinear absorption coefficient determined in this way results $\beta=1.1 \times 10^{-4} \mathrm{~cm} / \mathrm{W}$. Such analysis however does not fit the small humps visible in the pre-focal and post-focal regions.

The effect of Ar irradiation, on the other hand, is a dramatic change in the nonlinear absorption properties of the samples. As shown in Fig. 3b, in fact, the OA transmittance curve of the AuAg + $\mathrm{Ar}$ sample exhibits the superposition of two opposite features: a RSA component which induces the transmittance decrease visible in

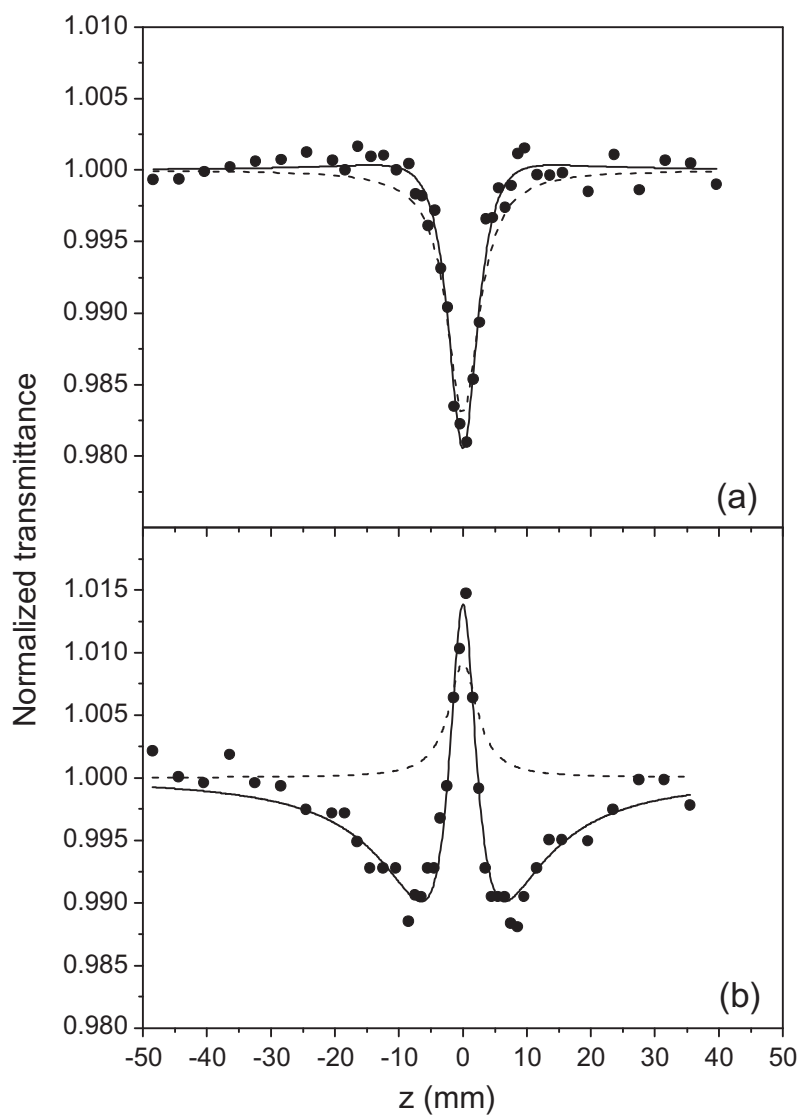

Fig. 4. Open aperture z-scan curves of the sample $\mathrm{AuAg}$ (a) and $\mathrm{AuAg}+\mathrm{Ar}$ (b), measured at $I_{0}=0.1 \mathrm{GW} / \mathrm{cm}^{2}$. The dashed lines are the best fits obtained in the Sheik-Bahae frame, while the solid lines are the best fits according to the method described in the text. the region far from the focus, and a SA component which gives rise to the peak in the region close to the focus. In this case, therefore, the conventional approach of fitting the data with Eq. (1) does not provide satisfactory results: the best fit obtained in this way is shown in Fig. $4 \mathrm{~b}$ (dashed line).

A much better agreement with the experimental data, instead, can be obtained by assuming an intensity-dependent nonlinear absorption coefficient in the form:

$\beta(I)=\frac{\beta_{1}}{1+\frac{I}{I_{s 1}}}+\frac{\beta_{2}}{1+\frac{I}{I_{s 2}}}$

where $\beta_{1}, \beta_{2}$ represent the effective nonlinear absorption coefficients related to RSA and SA processes (positive and negative, respectively), and $I_{s 1}, I_{s 2}$ are the corresponding saturation intensities. This expression can be introduced in the analytical formula given in Eq. (1) to fit the open aperture scans to determine the best values of the parameters $\beta_{1}, \beta_{2}, I_{s 1}, I_{s 2}$. The best fits obtained with this procedure are the solid lines reported in Fig. 4. By this method the OA scan of the AuAg + Ar sample is now well reproduced (Fig. 4b). Moreover, with this approach it is possible to fit also the small humps visible the OA scan of the AuAg sample (Fig. 4a) in the pre-focal and post-focal regions; these are indeed indicative of the presence also of a SA component which could not be taken into account with the conventional Sheik-Bahae approach. The numerical values are summarized in Table 1. Both samples exhibit similar large values of the SA and RSA coefficients. Nonetheless, the Ar irradiation, and consequently the NPls formation, seems to have a strong effect on the corresponding saturation intensities which resulted to be very different for the two systems. This is particularly important since it is the saturation intensity that controls the dominant behavior of these materials at high laser intensities, which changes from primarily RSA before Ar irradiation to SA after irradiation.The occurrence of SA and RSA processes at the same laser wavelength for different intensities has been observed in the past by Philip et al. in chemically synthesized AuAg nanoclusters in liquid solutions [11]. According to the authors, the ground-state plasmon bleaching is responsible for the SA behavior observed at moderate intensities; conversely, RSA is due to free carrier absorption and becomes significant for increased pump intensities. In an open aperture $z$-scan, these phenomena give rise to a transmittance increase in the pre-focal and post-focal regions (where the beam intensity impinging on the sample is low) followed by a decrease in the region close to the lens focus (where the beam intensity is maximum). The OA scan of sample AuAg (Fig. 4a) therefore can be interpreted in this framework. Conversely, the behavior of sample AuAg + Ar (Fig. 4b), in which RSA occurs in the low intensity region while SA is observed close to the focus, indicates that another process phenomenologically similar to SA is activated in this case. According to Philipp et al. a further change-over from RSA to SA would be expected in their samples at very high laser intensities. The fact that we observe this change-over in the Ar irradiated sample at low beam intensity (the same peak intensity used to measure the AuAg sample) could be therefore ascribed to the nanoplanets formation and to the local-field enhancement theoretically demonstrated in the satellite clusters halos in this sample [9]. Indeed, this could increase the effective intensity experienced by the material,

Table 1

Nonlinear absorption parameters of the investigated samples.

\begin{tabular}{lll}
\hline & AuAg & $\mathrm{AuAg}+\mathrm{Ar}$ \\
\hline$\beta_{1}(\mathrm{~cm} / \mathrm{W})$ & $(1.7 \pm 0.2) \times 10^{-4}$ & $(16 \pm 8) \times 10^{-4}$ \\
$I_{s 1}\left(\mathrm{~W} / \mathrm{cm}^{2}\right)$ & $(3.2 \pm 0.5) \times 10^{8}$ & $(1.0 \pm 0.4) \times 10^{7}$ \\
$\beta_{2}\left(\mathrm{~cm} / \mathrm{W}^{2}\right)$ & $(-3.42 \pm 0.06) \times 10^{-4}$ & $(-3.0 \pm 0.4) \times 10^{-4}$ \\
$I_{s 2}\left(\mathrm{~W} / \mathrm{cm}^{2}\right)$ & $(1.02 \pm 0.03) \times 10^{7}$ & $(1.21 \pm 0.05) \times 10^{9}$ \\
\hline
\end{tabular}


inducing the change-over from RSA to SA already at lower laser peak intensities. Nonetheless, the physical origin of the process inducing this further SA behavior is still not clear. Further investigations are in progress to clarify this aspect.

\section{Conclusions}

The nonlinear optical properties of Au-Ag nanoplanets in silica have been investigated by means of the z-scan technique. The results have shown that the formation of the nanoplanets due to Ar irradiation has a direct effect on the nonlinear absorption properties of the samples. In particular, local-field enhancement effects in the satellite cluster halos theoretically demonstrated in these systems could be responsible for the occurrence of the change-over from RSA to SA observed in the irradiated samples. A phenomenological function has been also developed to fit the open aperture zscan transmittance curves in order to quantitatively determine the nonlinear absorption parameters of these systems.

\section{References}

[1] P. Mazzoldi, G. Mattei, Synthesis of metal nanoclusters by using ion implantation, in: B. Corain, G. Schmid, N. Toshima (Eds.), Metal Nanoclusters in Catalysis and Materials Science the Issue of Size-Control, Elsevier, Amsterdam, 2007, p. 281.

[2] P. Mazzoldi, G. Mattei, Rivista del Nuovo Cimento 28 (2005) 1.

[3] G. Mattei, Nucl. Instrum. Meth. in Phys. Res. B 191 (2002) 323

[4] G. Mattei, P. Mazzoldi, H. Bernas, Metal nanoclusters for optical properties, in: H. Bernas (Ed.), Materials Science with Ion Beams, Springer-Verlag, Berlin, 2009.

[5] E. Cattaruzza, G. Battaglin, F. Gonella, G. Mattei, P. Mazzoldi, R. Polloni, B.F. Scremin, Appl. Surf. Sci. 247 (2005) 390.

[6] Y. Takeda, O. Plaksin, H. Wang, N. Kishimoto, Nucl. Instrn. and Meth. Phys. Res., Sect. B 257 (2007) 47.

[7] G. Mattei, G. De Marchi, C. Maurizio, P. Mazzoldi, C. Sada, V. Bello, G. Battaglin, Phys. Rev. Lett. 90 (2003) 085502

[8] V. Bello, G. De Marchi, C. Maurizio, G. Mattei, P. Mazzoldi, M. Parolin, C. Sada, J. Non-Cryst. Solids 345-346 (2004) 685.

[9] G. Pellegrini, V. Bello, G. Mattei, P. Mazzoldi, Opt. Express 15 (2007) 10097.

[10] M. Sheik-Bahae, A.A. Said, T.H. Wei, D.J. Hagan, E.W. Van Stryland, IEEE J. Quant. Electron. 26 (1990) 760.

[11] R. Philip, G. Ravindra Kumar, N. Sandhyarani, T. Pradeep, Phys. Rev. B 62 (2000) 13160. 